\title{
The Effect of Anode Configuration on Hydrogen MPD Thruster Performance: A Numerical Study
}

\author{
By Shitan TAUCHI, ${ }^{1)}$ Akira KAWASAKI, ${ }^{2)}$ Masakatsu NAKANE, ${ }^{3)}$ Kenichi KuBOTA, ${ }^{4)}$ and Ikkoh FUnAKI ${ }^{5)}$ \\ ${ }^{1)}$ Department of Space and Astronautical Science, SOKENDAI (The Graduate University for Advanced Studies), Sagamihara, Japan \\ ${ }^{2}$ Department of Aerospace Engineering, Nagoya University, Nagoya, Japan \\ ${ }^{3)}$ Department of Aerospace Engineering, Nihon University, Funabashi, Japan \\ ${ }^{4)}$ Aeronautical Technology Directorate, JAXA, Chofu, Japan \\ ${ }^{5}$ Institute of Space and Astronautical Science, JAXA, Sagamihara, Japan
}

(Received June 30th, 2017)

The flowfields of a self-field magnetoplasmadynamic (MPD) thruster using hydrogen propellant were numerically simulated with a physical model incorporating the ion-slip effect. Thrust performance was investigated for two anode configurations, namely, straight anode and flared anode at discharge currents between 5 to $8 \mathrm{kA}$. Simulation results show that thrust efficiency increases with increased discharge current for the straight anode, while for the flared anode, thrust efficiency tends to decrease; this opposite trend is caused by the ion-slip effect. When comparing thrust characteristics, thrust for the flared anode was found to be larger than that for the straight anode, but the advantage of the flared anode diminishes at higher discharge currents due to strong pinching and consequent pressure depletion in the vicinity of the flared anode surface. This pressure depletion leads to large electric power consumption owing to the ion-slip heating. That is, at lower pressures, the ion-slip effect becomes more significant because collisions between ions and neutral atoms are not frequent.

Key Words: Electric Propulsion, MPD Thruster, MHD, Hydrogen Plasma, Ion-slip

\begin{tabular}{lll} 
Nomenclature & \\
$\Delta V$ & $:$ velocity increment \\
$\boldsymbol{B}$ & $:$ magnetic induction vector \\
$\boldsymbol{E}$ & $:$ electric field vector \\
$E$ & $:$ energy per unit volume \\
$E_{t}$ & $:$ electric field along the boundary \\
$\boldsymbol{I}$ & $:$ unit tensor \\
$\boldsymbol{j}$ & $:$ current density vector \\
$J$ & $:$ discharge current \\
$\dot{m}$ & $:$ mass flow rate \\
$k$ & $:$ Boltzmann constant \\
$n_{s}$ & $:$ number density of chemical species $\mathrm{s}$ \\
$p$ & $:$ pressure \\
$Q_{i-j}$ & $:$ energy relaxation between $i$ and $j$ \\
$Q_{\text {diss }}^{s}$ & $:$ energy loss of heavy particles by \\
$Q_{\text {ion }}^{s}$ & $:$ energy loss of electron by ionization \\
$S_{\text {ion }}$ & $:$ ion slip parameter \\
$T$ & $:$ temperature \\
$t$ & $:$ time \\
$\boldsymbol{u}$ & $:$ mean speed vector \\
$\beta_{\mathrm{e}}$ & $:$ Hall parameter \\
$\lambda$ & $:$ heat conduction coefficient \\
$\mu_{0}$ & $:$ space permeability \\
$\rho$ & $:$ density \\
$\dot{\rho}$ & $:$ generation rate of chemical species s \\
$\sigma$ & $:$ electrical conductivity \\
\hline & & \\
\hline & & \\
\hline & &
\end{tabular}

$\begin{array}{lll}\tau & : \text { viscous stress tensor } \\ \alpha & : \text { ionization degree } \\ r & : \text { radius, radial coordinate } \\ z & : \text { axial coordinate } \\ F_{\text {total }} & : \text { total thrust } \\ F_{\text {mag }} & : \text { electromagnetic thrust } \\ F_{\text {aero }} & : \text { aerodynamic thrust } \\ F_{\text {th }} & : \text { theoretical electromagnetic thrust } \\ \eta & : \text { thrust efficiency } \\ r_{\text {in }} & : \text { inlet anode radius } \\ \theta & : \text { nozzle diverging angle } \\ P & : \text { input power } \\ P_{\text {Joule }} & : \text { power of joule heating } \\ P_{\text {lorentz }} & : \text { power of work by Lorentz } \\ P_{\text {ionslip }} & : \text { power of ion-slip } \\ V & : \text { discharge voltage } \\ V_{\text {sh }} & : \text { discharge voltage of sheath } \\ \text { Subscripts } & & \\ \text { a } & : \text { anode } \\ \mathrm{c} & : \text { cathode } \\ \mathrm{e} & : \text { electron } \\ \mathrm{h} & : \text { heavy particle } \\ \mathrm{i} & : \text { ion } \\ \mathrm{n} & : \text { neutral particle } \\ \text { tr } & : \text { translation } \\ \text { vib } & : \text { vibration } \\ \theta & : \text { azimuthal } \\ \end{array}$




\section{Introduction}

Nowadays, various kinds of manned Mars mission and other types of deep space missions are actively surveyed. ${ }^{1)}$ Most of these missions require both high $\Delta V$ and high payload ratio, that are possible only when high specific impulse thrusters are employed. One of the promising propulsion candidates for the main propulsion device of a large-scale deep space explorer will be magnetoplasmadynamic (MPD) thruster because of its high thrust density and high specific impulse.

This research deals with a steady-state, coaxial-geometry, self-field MPD thruster using hydrogen as a propellant. Figure 1 shows operational concepts of a self-field MPD thruster. The self-field MPD thruster ionizes a propellant and then accelerates a produced plasma not only aerodynamically but also electromagnetically by using a Lorentz force. Thrust of the self-field MPD thruster can be classified into electromagnetic thrust and aerodynamic thrust. The electromagnetic thrust is equivalent to the Lorentz force produced by the product of a discharge current and an induced magnetic field. The aerodynamic thrust corresponds to a force acting on the thruster channel, which is equivalent to pressure of high temperature gas heated by plasma discharge.

Self-field MPD thrusters have not been applied to actual missions yet because of low technological readiness. There are several issues to be solved, for example, enormous input power and thermal problem of the electrode, ${ }^{2)}$ and improvement of thrust efficiency, which contributes to the reduction of thermal loss, should be attained before practical use. Thus, this research focused particularly on improvement of thrust efficiency. As a propellant, hydrogen was selected because it is widely known that it can attain the highest thrust efficiency among various gas species due to the large contribution of aerodynamic thrust. ${ }^{3,4)}$ Since hydrogen is a molecular gas, dissociation process should occur before ionization, thus electrical conductivity tends to be high only at a downstream region. This causes extension of current paths toward the downstream region, and then results in high discharge voltages. Numerical simulation should also consider the ion-slip effect since the previous studies revealed that the ion-slip, ${ }^{5-12)}$ and the selection of anode configuration are significantly important for hydrogen MPD thrusters. ${ }^{13-17)}$ In this study, several thruster geometries are selected and simulated for different nozzle diverging angles to clarify the effects of the anode configuration on the thrust performance. Also, importance of the ion-slip effect is discussed based on some analyses of numerical simulation results.

\section{Numerical Modeling}

Assumptions in this study were shown below.

The plasma flow was treated as a magnetohydrodynamic (MHD) flow.

- The flow and electromagnetic fields were axisymmetric.

- Considered chemical species were hydrogen molecule $\left(\mathrm{H}_{2}\right)$, hydrogen atom $(\mathrm{H})$, hydrogen ion $\left(\mathrm{H}^{+}\right)$, and electron $\left(\mathrm{e}^{-}\right)$.

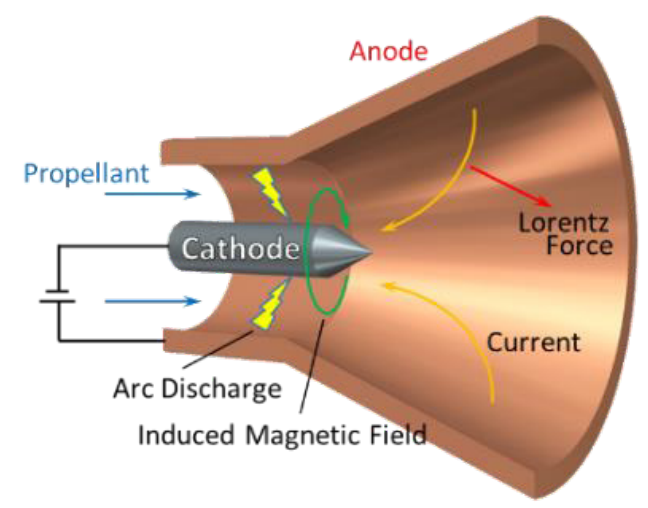

Fig. 1. Operational concept of an MPD thruster.

- The flow was laminar.

- Thermal conductivity and viscosity were considered.

- Three-temperature (translational temperature, vibrational temperature, and electron temperature) nonequilibrium was considered. An upper limit was specified for the translational and vibrational temperatures.

- The Hall effect and ion-slip effect were considered.

The basic equations follow a standard resistive MHD approximation with some additional equations which are described below: ${ }^{7,8,10,11)}$

Conservation equation of total mass:

$$
\frac{\partial \rho}{\partial t}+\nabla \cdot(\rho \mathbf{u})=0
$$

Conservation equation of atom and ion masses:

$$
\frac{\partial \rho_{s}}{\partial t}+\nabla \cdot\left(\rho_{s} \mathbf{u}\right)=\dot{\rho} \quad\left(s=H_{2}, H, H^{+}\right)
$$

Navier-Stokes equation coupled with Lorentz force:

$$
\frac{\partial \rho \mathbf{u}}{\partial t}+\nabla \cdot(\rho \mathbf{u} \mathbf{u}+p \mathbf{I})=\mathbf{j} \times \mathbf{B}+\nabla \cdot \boldsymbol{\tau}
$$

Conservation equation of heavy particle energy:

$$
\begin{aligned}
& \quad \frac{\partial E_{h}}{\partial t}+\nabla \cdot\left[\left(E_{h}+p\right) \mathbf{u}\right] \\
& =p_{e} \nabla \cdot \mathbf{u}+\mathbf{u} \cdot(\mathbf{j} \times \mathbf{B})+\frac{S_{\text {ion }}}{\sigma|\mathbf{B}|^{2}}(\mathbf{j} \times \mathbf{B})^{2}+\nabla \cdot\left(\lambda_{v i b} \nabla T_{v i b}\right) \\
& +\nabla \cdot\left\{\left(\lambda_{t r}+\lambda_{r o t}\right) \nabla T_{t r}\right\}+\nabla \cdot(\boldsymbol{\tau} \mathbf{u})-Q_{t r-e}+Q_{e-v i b}-Q_{d i s s}^{h} \\
& \text { Conservation equation of electron energy: } \\
& \quad \frac{\partial E_{e}}{\partial t}+\nabla \cdot\left(E_{e} \mathbf{u}\right)=-p_{e} \nabla \cdot \mathbf{u} \\
& \quad+\nabla \cdot\left(\lambda_{e} \nabla T_{e}\right)+\frac{\mathbf{j}^{2}}{\sigma}+Q_{t r-e}-Q_{e-v i b}-Q_{d i s s}^{e}-Q_{i o n}^{e}
\end{aligned}
$$

Conservation equation of molecular vibrational energy:

$$
\begin{aligned}
\frac{\partial E_{v i b}}{\partial t}+\nabla \cdot\left(E_{v i b} \mathbf{u}\right)= & \nabla \cdot\left(\lambda_{v i b} \nabla T_{v i b}\right) \\
& +Q_{t r-v i b}+Q_{e-v i b}-Q_{d i s s}^{e}-Q_{i o n}^{h}
\end{aligned}
$$

Generalized Ohm's law:

$$
\mathbf{j}=\sigma(\mathbf{E}+\mathbf{u} \times \mathbf{B})-\frac{\beta_{e}}{|\mathbf{B}|} \mathbf{j} \times \mathbf{B}+\frac{S_{i o n}}{|\mathbf{B}|^{2}}(\mathbf{j} \times \mathbf{B}) \times \mathbf{B}
$$

Equation of state:

$$
p=\sum_{s} n_{s} k T_{t r}+n_{e} k T_{e} \quad\left(s=H_{2}, H, H^{+}\right)
$$


Induction equation:

$$
\begin{aligned}
& \frac{\partial \mathbf{B}}{\partial t}-\nabla \times(\mathbf{u} \times \mathbf{B}) \\
& =-\nabla \times\left\{\frac{1}{\mu_{0} \sigma} \nabla \times \mathbf{B}+\frac{1}{\mu_{0} e n_{e}}(\nabla \times \mathbf{B}) \times \mathbf{B}\right\} \\
& +\nabla \times\left[\frac{S_{\text {ion }}}{\mu_{0} \sigma|\mathbf{B}|^{2}}\{(\nabla \times \mathbf{B}) \times \mathbf{B}\} \times \mathbf{B}\right]
\end{aligned}
$$

In the above equations, evaluation formula of Ion-slip parameter is used:

$$
S_{\text {ion }}=(1-\alpha)^{2} \beta_{e} \beta_{h}
$$

\section{Numerical Analysis}

\subsection{Numerical method}

Numerical analysis method and analysis scheme were described in detail in reference, ${ }^{7)}$ thus will be briefly summarized here. The basic equations were discretized in generalized coordinate, and were solved with the time-marching method. In evaluation of the convection term, the HLL (Harten-Lax-van Leer) scheme was used with second-order MUSCL interpolation, where TVD (total variation diminishing) condition was imposed with the minmod function. The second derivatives were discretized with the central-difference scheme. In the time integration, the first-order Euler explicit method was used in order to accelerate the convergence of calculation, and the point implicit method was applied to the diffusion term of magnetic field.

These calculations were performed on JAXA Supercomputer System Generation 2 (JSS2).

\subsection{Calculation conditions}

Table 1 shows the calculation conditions. Since a constant current power supply was used in the experiment, the discharge current was specified as an input parameter. Then the induced azimuthal magnetic flux density at the inlet could be evaluated from the Ampère's law.

$$
B_{\theta, \text { in }}=-\frac{\mu_{0} J}{2 \pi r}
$$

The equipotential condition along the electrodes was given by the following condition; the electric field along the boundary $E_{t}$ was set at zero. (Table 1)

\subsection{Configuration parameters}

In this research, the flows for two nozzle diverging angles were simulated. The other configuration parameters are shown in Table 2 and Fig. 2.

\section{Numerical Results and Discussion}

This section shows the effect of the nozzle diverging angle on the thrust performance. Equations from (12) to (16) show the definitions of the aerodynamic thrust, electromagnetic thrust, discharge voltage, thrust efficiency and consumption power, respectively. The total thrust was sum of the

\begin{tabular}{|c|c|}
\hline Propellant & $\mathrm{H}_{2}$ \\
\hline Discharge current $J, \mathrm{kA}$ & $5,5.5,6,6.5,7,7.5,8$ \\
\hline Mass flow rate $\dot{m}, \mathrm{~g} / \mathrm{s}$ & 0.4 \\
\hline $\begin{array}{l}\text { Inlet electron } \\
\text { temperature, } \mathrm{K}\end{array}$ & 600 \\
\hline $\begin{array}{l}\text { Inlet heavy particle } \\
\text { temperature, } \mathrm{K}\end{array}$ & 600 \\
\hline $\begin{array}{l}\text { Inlet dissociation } \\
\text { degree, - }\end{array}$ & $1.0 \times 10^{-2}$ \\
\hline Inlet ionization degree, - & $1.0 \times 10^{-3}$ \\
\hline $\begin{array}{l}\text { Wall condition of } \\
\text { flow-field }\end{array}$ & Limited isothermal condition \\
\hline $\begin{array}{c}\text { Anode wall } \\
\text { temperature, } \mathrm{K}\end{array}$ & $\begin{array}{l}T_{v i b}, T_{t r}<1300: \text { adiabatic condition } \\
T_{v i b}, T_{t r} \geqq 1300: \text { isothermal condition } \\
T_{e}: \text { Calculate from adiabatic condition }\end{array}$ \\
\hline $\begin{array}{l}\text { Cathode wall } \\
\text { temperature, } \mathrm{K}\end{array}$ & $\begin{array}{l}T_{v i b}, T_{t r}<2000: \text { adiabatic condition } \\
T_{v i b}, T_{t r} \geqq 2000 \text { : isothermal condition } \\
T_{e}: \text { Calculate from adiabatic condition }\end{array}$ \\
\hline Sheath voltage, $\mathrm{V}$ & 20 \\
\hline $\begin{array}{l}\text { Equipotential condition } \\
\text { along the electrodes }\end{array}$ & $E_{t}=0$ \\
\hline
\end{tabular}
aerodynamic thrust and the electromagnetic thrust. Both power of the joule heating and power of the ion-slip heating were evaluated propellant heating. Figure 3 shows the
Table. 1. Analysis and calculation conditions.

Table. 2. Configuration parameters.

Inlet anode radius $r_{\text {in }}, \mathrm{mm} \quad 40$

Nozzle diverging angle $\theta$, deg $\quad 0,35$

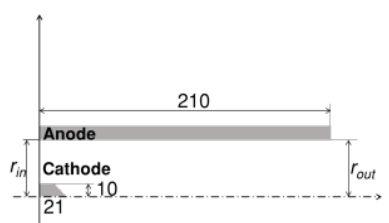

(a) Straight anode $\left(\theta=0^{\circ}\right)$

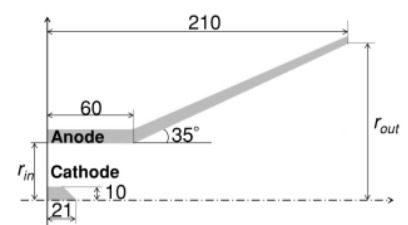

(b) Flared anode $\left(\theta=35^{\circ}\right)$
Fig. 2. Configuration parameters schematic. [mm]

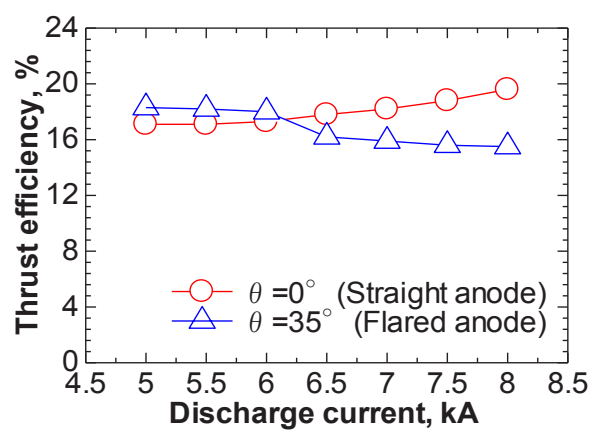

Fig. 3. Discharge current vs. thrust efficiency.

relationship between the thrust efficiency and the discharge current. The thrust and the specific impulse are also shown in Fig. 4.

Aerodynamic thrust:

$$
\begin{aligned}
F_{\text {aero }} & =\iint_{\text {Inlet }}\left[p+\rho\left(\mathbf{u} \cdot \mathbf{e}_{z}\right)^{2}\right] \mathbf{e}_{z} \cdot d \mathbf{S} \\
& +\iint_{\text {Anode-sidewall }}\left(p-\mathbf{e}_{r} \cdot \boldsymbol{\tau} \cdot \mathbf{e}_{z}\right) \mathbf{e}_{z} \cdot d \mathbf{S}
\end{aligned}
$$

Electromagnetic thrust:

$$
\begin{aligned}
F_{\text {mag }}= & \iiint\left[(\mathbf{j} \times \mathbf{B}) \cdot \mathbf{e}_{z}\right] d V \\
& +\underset{\text { Cathode sidewall }}{\iint\left(p-\mathbf{e}_{r} \cdot \boldsymbol{\tau} \cdot \mathbf{e}_{z}\right) \mathbf{e}_{z} \cdot d \mathbf{S}}
\end{aligned}
$$

Discharge voltage:

$$
V=\frac{\int \mathbf{j} \cdot \mathbf{E} d v}{J}+V_{s h} \quad\left(V_{s h}=20 V\right)
$$




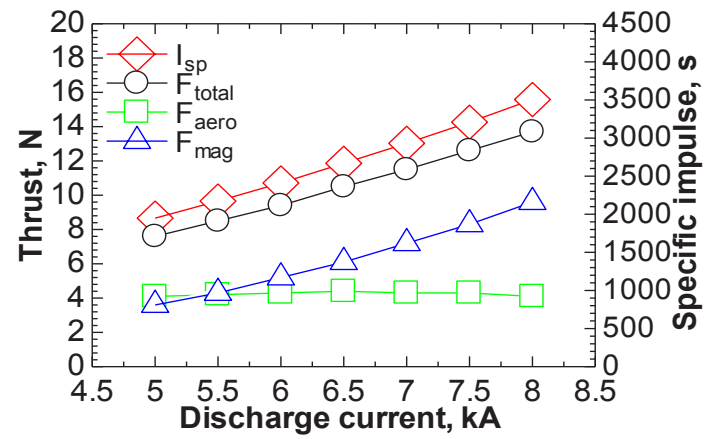

(a) Straight anode

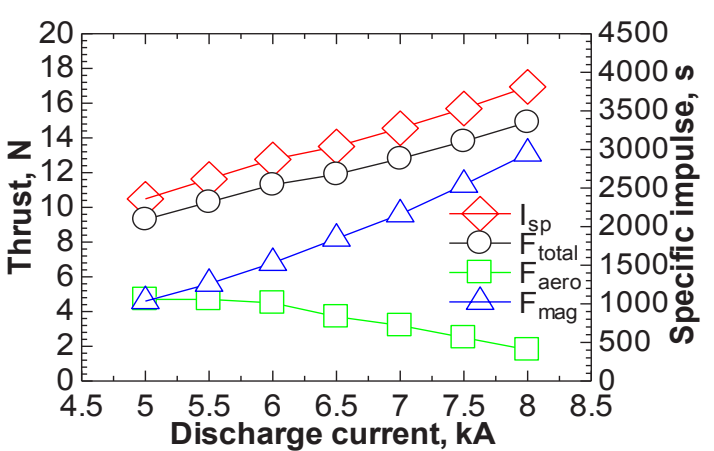

(b) Flared anode

Fig. 4. Discharge current vs. thrust.

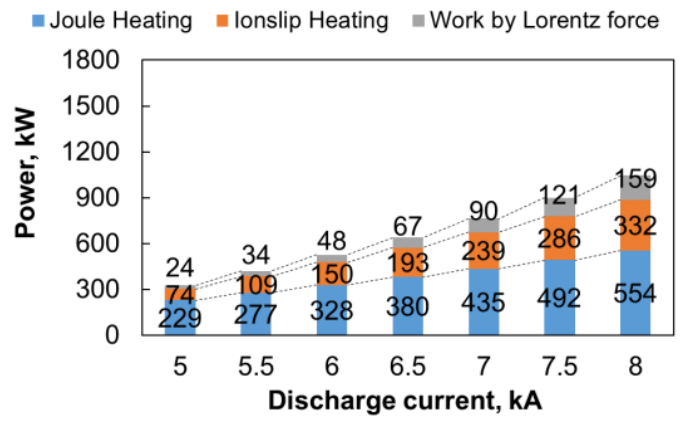

(a) Straight anode

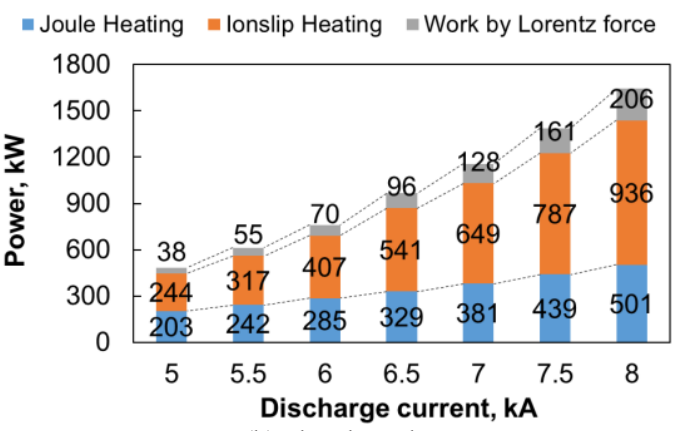

(b) Flared anode

Fig. 5. Discharge current vs. discharge energy breakdown.

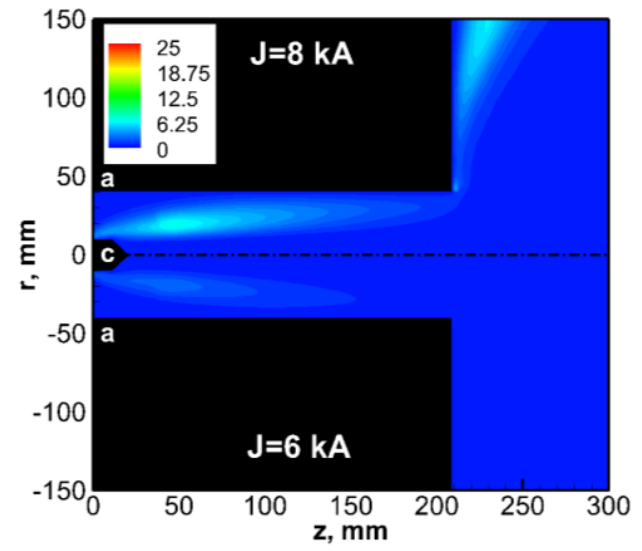

(a) Straight anode

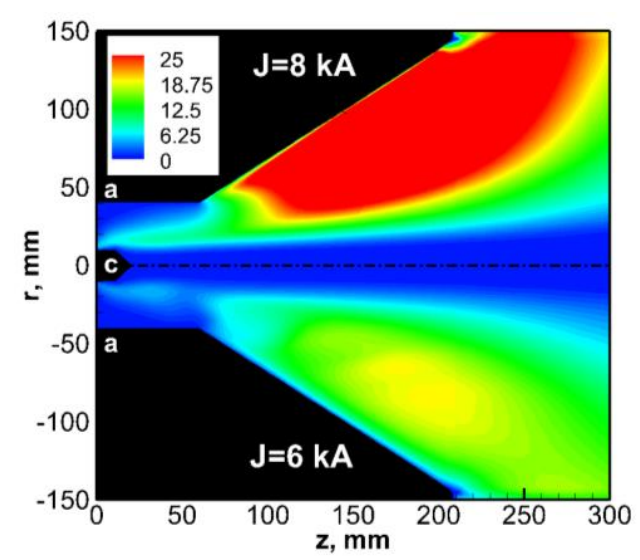

(b) Flared anode

Fig. 6 . Ion-slip parameter (color). (Lower half: $J=6 \mathrm{kA}$, upper half: $J=8 \mathrm{kA}$ )

Thrust efficiency:

$$
\eta=\frac{\dot{m} u_{e}^{2}}{2 P}=\frac{F_{\text {total }}^{2}}{2 \dot{m} P}=\frac{F_{\text {total }}^{2}}{2 \dot{m} J V}
$$

Power:

$$
\begin{aligned}
\mathbf{P} & =P_{\text {joule }}+P_{\text {lorentz }}+P_{\text {ionslip }} \\
& =\int\left(\frac{j^{2}}{\sigma}+\mathbf{u} \cdot(\mathbf{j} \times \mathbf{B})+\frac{j^{2}}{\sigma} S_{\text {ion }}\right) d V
\end{aligned}
$$

Figure 3 indicates how thrust efficiency depends on nozzle diverging angles. Thrust efficiency increased with increasing discharge current for the straight anode $\left(\theta=0^{\circ}\right)$, but for the flared anode $\left(\theta=35^{\circ}\right)$, thrust efficiency decreased with increasing discharge current. To understand this reverse trend, thrust and power consumption are plotted against discharge current in Figs. 4 and 5. According to Fig. 4, the increasing rate of thrust for current in the case of flared anode was comparable with that of the straight anode in spite of the large discrepancy of electromagnetic fields. This is because the aerodynamic thrust was rapidly decreased with current. On the other hand, according to Fig. 5, the growth rate of the power consumption against current in the case of the flared anode was higher than that of straight anode due to the significant increase in power due to the ion-slip effect. Large power consumption of the flared anode will be the reason for low thrust efficiency in the high discharge current regime. It should be also noted that in the low discharge current regime, the flared anode is more efficient than the straight anode.

Figures 6-8 shows the ion-slip parameter distribution, the Hall parameter distribution, and the electric power consumption regarding the ion-slip in cases of straight/flared anode for $J=6$ and $8 \mathrm{kA}$. In Fig. 7, the current path was overlapped, and sonic line was overlapped in Fig. 8. Figure 6 (b) shows the ion-slip parameter for the flared anode significantly large near the flared anode surface, which is due 


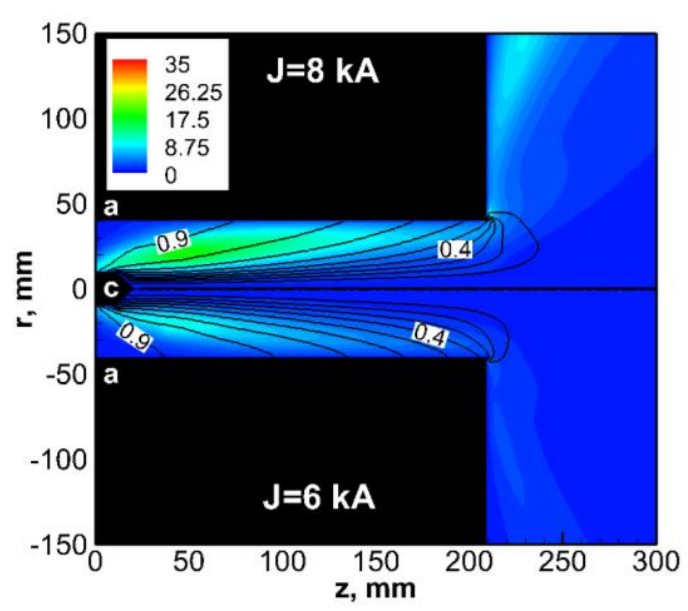

(a) Straight anode

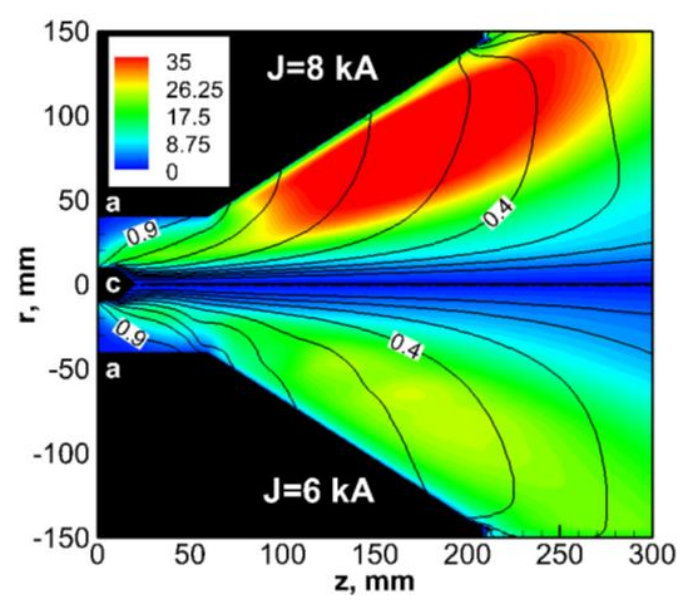

(b) Flared anode

Fig. 7. Hall parameter (color) and current path (lines). (Lower half: $J=6 \mathrm{kA}$, Upper half: $J=8 \mathrm{kA}$ )

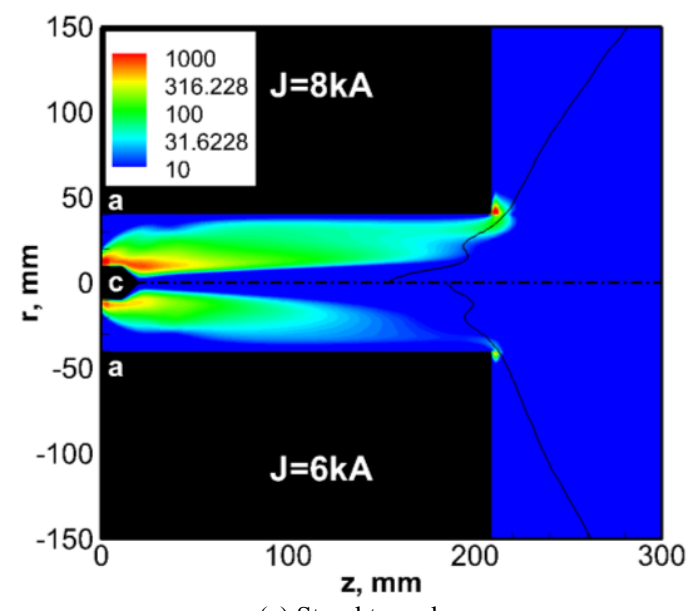

(a) Straght anode

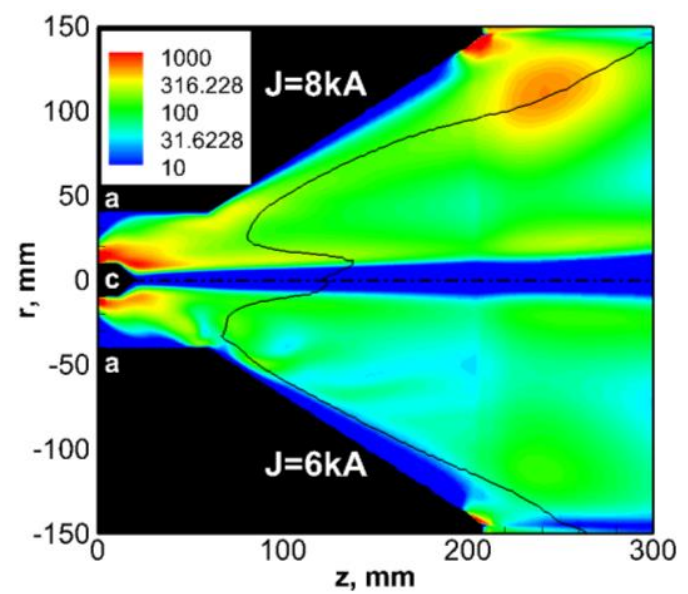

(b) Flared anode

Fig. 8 . Power consumption by the ion-slip heating (color) and sonic lines. (Lower half: $J=6 \mathrm{kA}$, Upper half: $J=8 \mathrm{kA}$ )

to depletion of the propellant. The reason for this was related to the extended current path as shown in Fig. 7 (b). Since the current path was extended toward the downstream region due to high Hall parameter, the flow was constricted toward the central axis. This flared anode case results in larger power consumption regarding the ion-slip than that for the straight anode near the anode surface, which means the plasma was heated strongly in this area. In spite of the heating, however, the power was not converted to the aerodynamic thrust effectively. The reason for this was related to location of power consumption as shown in Fig. 8 (b). Since the ion-slip power consumption occurred in a supersonic region, the power was not converted effectively to the aerodynamic thrust.

\section{Conclusion}

To clarify the effects of anode configuration on the performance of a self-field MPD thruster using hydrogen as the propellant, a straight anode and a flared anode were compared by numerical simulation, varying the discharge current from 6 to $8 \mathrm{kA}$. The mass flow rate was fixed to 0.4 $\mathrm{g} / \mathrm{s}$.

The results show that thrust efficiency was increased with increasing current due to enhanced electromagnetic force when the nozzle diverging angle was sufficiently small (straight anode, $\theta=0^{\circ}$ ). On the other hand, when the nozzle diverging angle was large (flared anode, $\theta=35^{\circ}$ ), a reverse correlation was found between the discharge current and the thrust efficiency although the increase in the electromagnetic force. This is due to rarefaction of the flow in the vicinity of the flared anode surface and consequent large power consumption due to ion-slip heating. When the nozzle diverging angle was large, although the plasma was highly heated via the ion-slip in the downstream of the discharge channel, heated power was not converted efficiently to aerodynamic thrust because power is deposited at the supersonic area and hence effective energy conversion to aerodynamic thrust is prevented.

Upon the actual design of a self-field hydrogen MPD thruster, the designer should make the anode having sufficiently small nozzle diverging angle in order to reduce the ion-slip heating, particularly at high discharge currents. When an MPD designer considers development of low discharge current level thruster, they should make the anode having excessively large diverging angle nozzle in order to optimize the nozzle effect. 


\section{References}

1) ISECG: The Global Exploration Roadmap, NASA, 2013

2) Kuriki, K. and Arakawa, Y.: Introduction to Electric Propulsion, University of Tokyo Press, 2003, pp. 119-139 (in Japanese).

3) Tahara, H., Sakakibara, T., Kagaya, Y. and Yoshikawa, Y.: Operational Characteristics and Discharge Mechanisms of Quasisteady Magnetoplasmadynamic (MPD) Arjet for Space Propulsion, Res. nuclear fusion, 64 (1990), pp. 119-139 (in Japanese)

4) Uematsu, K., Morimoto, S. and Kuriki, K.: MPD Thruster Performance with Various Propellants, J. Space. Rockets, 22 (1985), pp.412-416

5) Malliaris, A. C. and Libby, D. R.: Spectroscopic study of ion-neutral coupling in plasma acceleration, AIAA J., 9 (1970), pp. 160-168.

6) Mitchner, M. and Kruger, C. H., Jr.: Partially Ionized Gases, John Wiley \& Sons, Inc., 1973, pp. 173-182.

7) Sato, H., Kubota, K. and Funaki, I..: Modeling and Numerical Simulation of a Two-Dimensional MPD thruster using a Hydrogen Propellant, Proc. 32nd IEPC, IEPC-2011-258, 2011.

8) Tauchi, S., Nakane, M., Kawasaki, A., Kubota, K, and Funaki, I.: Numerical Analysis on Anode Shape Dependence of Hydrogen MPD Thruster Performance, Proc. Space Sci. Tech. Conference, 60 (2016), JSASS-2016-4642. (in Japanese).

9) Kinefuchi, K., Funaki, I., Toki, K. and Shimizu, Y.: Measurement of Velocity and Power Balance in a Two-Dimensional MPD Arcjet, J. Jpn. Soc. Aeronaut. Space Sci., 53 (2005), pp. 215-223. (in Japanese)

10) Kajikawa, Y., Nakane, M., Ishikawa, Y., Kubota, K. and Funaki,
I.: Nozzle Shape Optimization of a Hydrogen MPD Thruster using the Isothermal Wall Mode, Proc. Space Sci. Tech. Conference, 59 (2015), JSASS-2015-4271. (in Japanese).

11) Kawasaki, A., Kubota, K., Funaki, I. and Okuno, Y.: Numerical Investigation of Discharge Current Path in a Hydrogen MPD Thruster, IEEJ Trans. Fundament. Materials, 136 (2016), pp. 141-146 (in Japanese).

12) Mikellides, P. G.: Modeling and Analysis of a Megawatt-Class Magnetoplasmadynamic Thruster, J. Propul. Power, 20 (2004), pp. 204-210.

13) Heimerdinger, D. J. and Martinez-Sanchez, M.: Design and Performance of an Annular Magnetoplasmadynamic Thruster, $J$. Propul. Power, 7 (1991), pp.975-980.

14) Paccani, G. and Petrucci, L. : Experimental Analysis of a Solid Propellant MPD Thruster with Different Anode Radii, Proc. 32nd IEPC, IEPC-99-235, 1991, pp.1370-1377.

15) Nakata, D., Toki, K., Funaki, I., Shimizu, Y., Kuninaka, H. and Arakawa, Y.: Geometric Parameter Effect of Magneto-Plasma-Dynamic Thruster, IAC-05-IAF-E.2.11, 2005.

16) Nakane, M., Nagao, M., Ishikawa, Y., Kubota, K. and Funaki, I.: Nozzle Shape Optimization for an MPD Thruster Using a Two-Dimensional Axisymmetric Flow Model, J. Jpn. Soc. Aeronaut. Space Sci., 59 (2011), pp.90-96. (in Japanese)

17) Malliaris, A. C., John, R. R., Garrison, R. L. and Libby, D. R.: Performance of Quasi-Steady MPD Thrusters at High Powers, AIAA J., 10 (1972), pp. 121-122. 